\title{
Continuous Operations and Series of Indeterminate Terms
}

\section{Eduardo Diedrich}

Independent Researcher, Salta, Argentina

Email: eduardo.diedrich@outlook.com.ar

How to cite this paper: Diedrich, E. (2021) Continuous Operations and Series of Indeterminate Terms. Journal of Applied Mathematics and Physics, 9, 2215-2223. https://doi.org/10.4236/jamp.2021.99141

Received: July 3, 2021

Accepted: September 11, 2021

Published: September 14, 2021

Copyright (c) 2021 by author(s) and Scientific Research Publishing Inc. This work is licensed under the Creative Commons Attribution International License (CC BY 4.0).

http://creativecommons.org/licenses/by/4.0/

\begin{abstract}
It is known that functions involving natural numbers are generalized to the real ones, for instance the gamma function can be viewed as a generalization of the factorial operator. In this paper, we propose to generalize the repetition of an operation over a function (composition, derivatives and integrals) toward the field of reals. It means repeating $\mathrm{q}$ times an operation over a function, where $\mathrm{q}$ is a real number. As a result, it is explained what functional and analytical dimensional extensions are and it is given a proof to theorems related to the indeterminate terms. The main finding is that every real number is expressible as a bijection of an infinite sum of elements whose coefficients are real numbers and their main values are either an indeterminate value or an infinite value. The concept of series of indeterminate values becomes relevant, as a novelty to operate with infinite, zero and indeterminate terms, which cannot be deductible from the non-standard analysis.
\end{abstract}

\section{Keywords}

Continuous Derivative, Analytical-Dimensional Extension, Series of Indeterminate Values

\section{Introduction}

The fact in Mathematical Analysis of the supposed impossibility of operating with indeterminate terms is known. The following article introduces a series of concepts that allow formulating and proving theorems that states that under certain conditions, operations with indeterminate terms give an ordinary real value. In the study of calculus, some derivation and integration methods are learned, as well as the great importance that these mathematical tools have in science and engineering. This is what we know as ordinary or integer integral and differential calculus. Here some questions arise: Why should $\mathrm{n}$ be $1,2,3$ ? Is 
there a possibility that $\mathrm{n}$ is a real number? [1]. Mathematicians who have proposed different definitions for the fractional calculation (keeping in mind $\mathrm{n}$ as a fraction) have been, among others: Lacroix, Euler, Riemann, Caputo etc., each with their own reasoning [1] [2]. In this work, we focus on generalizing the derivative of a power function to the field of reals and taking as reasoning that there is a set of mathematical functions expressible as an infinite sum of power functions, by means of the distributive property of the derivative with respect to the sum, we generalize the concept of continuous derivative to developable functions as power series. Concepts like dimensional analytical extension arise naturally. Then we use the sine function, because it is developable as a series of powers and at the same time, due to its properties of periodicity and complementarity with the cosine function, it has an extra generalization for its development as a continuous derivative. This double definition for the sine function is a bridge that allows us to arrive at the quasi-paradoxical result of the ind-series (series of zero, indeterminate and infinite terms).

\section{The Continuous Derivative Operator}

\subsection{The Exponential Function}

Let $F(x)=\mathrm{e}^{k x}$ where $k \in \mathbb{R}$ and $F: \mathbb{R} \rightarrow \mathbb{R}$. Then

$$
\begin{aligned}
& \frac{\partial^{1} F(x)}{\partial x^{1}}=k \mathrm{e}^{k x} \\
& \frac{\partial^{2} F(x)}{\partial x^{2}}=k^{2} \mathrm{e}^{k x} \\
& \frac{\partial^{i} F(x)}{\partial x^{i}}=k^{i} \mathrm{e}^{k x}
\end{aligned}
$$

We can generalize the repetition of derivative operator to the field of reals

$$
\frac{\partial^{q} F(x)}{\partial x^{q}}=k^{q} \mathrm{e}^{k x}
$$

where $q \in \mathbb{R}$.

\subsection{The Power Function}

Let $F(x)=x^{k}$ where $k \in \mathbb{R}$ and $F: \mathbb{R} \rightarrow \mathbb{R}$. Then

$$
\begin{gathered}
\frac{\partial^{1} F(x)}{\partial x^{1}}=k x^{k-1} \\
\frac{\partial^{2} F(x)}{\partial x^{2}}=k(k-1) x^{k-2}=\frac{k !}{(k-2) !} x^{k-2} \\
\frac{\partial^{i} F(x)}{\partial x^{i}}=\frac{k !}{(k-i) !} x^{k-i}
\end{gathered}
$$

We can generalize the repetition of derivative operator to the field of reals

$$
\frac{\partial^{q} F(x)}{\partial x^{q}}=\frac{k !}{(k-q) !} x^{k-q}
$$


where $q \in \mathbb{R}$

$$
\frac{\partial^{q} F(x)}{\partial x^{q}}=\frac{k !}{(k-q) !} x^{k-q}=\frac{\Gamma(k+1)}{\Gamma(k-q+1)} x^{k-q}
$$

So

$$
(x, q)^{k}=\frac{k !}{(k-q) !} x^{k-q}=\frac{\Gamma(k+1)}{\Gamma(k-q+1)} x^{k-q}
$$

\section{Functional and Analytical Dimensional Extensions}

\subsection{Functional Extension}

Let $F(x)$ such that $F: \mathbb{R} \rightarrow \mathbb{R}$ then: $F_{e}(x, y)$ such that $F_{e}: \mathbb{R}^{2} \rightarrow \mathbb{R}$ is a functional extension of $F(x)$ if: there exists at least one value of $y$, whereby it is true that:

$$
F_{e}\left(x, y=y_{i}\right)=F(x)
$$

\subsection{Analytical Dimensional Extension}

As a consequence of the expressed above, let $F(x)$ be continuously operable by an integral or derivative operator along $\mathbb{R}$.

In case of the derived function, we have $F_{a}(x, q)=\frac{\partial^{q} F(x)}{\partial x^{q}}$.

So $F_{a}(x, q)$ is a special case of functional extension, that we can call analytical dimensional extension of $F(x)$.

It is true that $F_{a}(x, q=0)=F(x)$.

\section{Application of the Concept to Another Functions}

\subsection{Development in Power Series}

Note that

$$
\frac{\partial^{q} F+G(x)}{\partial x^{q}}=\frac{\partial^{q} F(x)}{\partial x^{q}}+\frac{\partial^{q} G(x)}{\partial x^{q}}
$$

So, it is followed that all function expressible by a power series have real derivative, and consequently a-d extension.

\subsection{The Sine Function}

Using the Taylor development formula, we can express the sine function as follows:

$$
\begin{gathered}
F(x)=\sin (x)=\lim _{n \rightarrow \infty} \sum_{i=0}^{n}(-1)^{i} \frac{x^{2 i+1}}{(2 i+1) !} \\
\frac{\partial^{q} \sin (x)}{\partial x^{q}}=\lim _{n \rightarrow \infty} \sum_{i=0}^{n}(-1)^{i} \frac{\frac{\Gamma(2 i+2)}{\Gamma(2 i+2-q)} x^{2 i+1-q}}{\Gamma(2 i+2)}
\end{gathered}
$$




$$
\frac{\partial^{q} \sin (x)}{\partial x^{q}}=\lim _{n \rightarrow \infty} \sum_{i=0}^{n}(-1)^{i} \frac{x^{2 i+1-q}}{\Gamma(2 i+2-q)}
$$

On the other hand, we use another generalization, trough the following basic property:

$$
\frac{\partial^{q} \sin (x)}{\partial x^{q}}=\sin \left(x+\frac{\pi}{2} q\right)
$$

So

$$
\sin (x, q)=\sin \left(x+\frac{\pi}{2} q\right)
$$

Therefore

$$
\begin{aligned}
& \sin (x)=\sin (x, 0)=\sin \left(x+\frac{\pi}{2} 0\right)=\sin \left(0+\frac{\pi}{2} x \frac{2}{\pi}\right)=\sin \left(0, \frac{2 x}{\pi}\right) \\
& \lim _{n \rightarrow \infty} \sum_{i=0}^{n}(-1)^{i} \frac{x^{2 i+1}}{\Gamma(2 i+2)}=\lim _{n \rightarrow \infty} \sum_{i=0}^{n}(-1)^{i} \frac{0^{2 i+1-\frac{2 x}{\pi}}}{\Gamma\left(2 i+2-\frac{2 x}{\pi}\right)}
\end{aligned}
$$

At the right side of the equality we observe that appears $0^{a}$, that in some cases could be equal to $\infty$, but by intuition we try to avoid a situation where the series term were indeterminate or infinite.

Concepts such as infinite or infinitesimal are based on the hyper-real numbers. The infinitesimals would be numbers smaller than any conventional real number, and their respective inverses would correspond to "infinite" or "unbounded" numbers.

Therefore:

If $2 i+1-\frac{2 x}{\pi}<0$ then $\frac{1}{\Gamma\left(2 i+2-\frac{2 x}{\pi}\right)}=\infty$ and consequently $\Gamma\left(2 i+2-\frac{2 x}{\pi}\right)=0$.

But by its own definition the $\Gamma$ function never equals zero.

Consider for one instance that $\infty$ is a number. Then we have a series with terms whose values are zeroes, infinite and minus infinite and the series equals a real ordinary number.

Let's call them the ind-series or $I S(x)$. As sin function takes values in the $[-1,1]$ interval, and the same is bijectable to the $\mathbb{R}$ numbers (through a reciprocal function, for example). Then we are in conditions to prove some theorems:

\section{Formal Proof}

Definition 1 (Continuous Derivative of power function).

Be $F(x)=x^{k}$

$$
\frac{\partial^{q} F(x)}{\partial x^{q}}=\frac{\Gamma(k+1)}{\Gamma(k-q+1)} x^{k-q}
$$


where $q \in \mathbb{R}$. Is the Continuous Derivative of $F(x)$ of $q$ order.

Lemma 1 (Continuous Derivative of a function developable by power series).

Be $F(x)$ developable by power series as $\lim _{n \rightarrow \infty} \sum_{i=0}^{n} a_{i}^{i} x^{i}$.

$$
\frac{\partial^{q} F(x)}{\partial x^{q}}=\lim _{n \rightarrow \infty} \sum_{i=0}^{n} a_{i}^{i} \frac{\Gamma(i+1)}{\Gamma(i-q+1)} x^{i-q}
$$

where $q \in \mathbb{R}$ and $a_{i} \in \mathbb{R}$. And it is called the Continuous Derivative of $F(x)$ of $q$ order.

Proof of Lemma 1.

Using the distributive property of the derivative operator respect to the sum of functions and the Definition 1, the lemma is proved.

Lemma 2 (Symmetry of values of sine function).

Be $F(x)=\sin (x)$

$$
\begin{aligned}
\sin \left(\frac{\pi}{2}-x\right) & =\sin \left(\frac{\pi}{2}+x\right) \\
\sin \left(\frac{3 \pi}{2}-x\right) & =\sin \left(\frac{3 \pi}{2}+x\right)
\end{aligned}
$$

Proof of Lemma 2.

Using the properties of the sine function is proved.

$$
\begin{gathered}
\sin \left(\frac{\pi}{2}-x\right)=\sin \left(\frac{\pi}{2}\right) \cos (x)-\cos \left(\frac{\pi}{2}\right) \sin (x) \\
\sin \left(\frac{\pi}{2}-x\right)=\cos (x) \\
\sin \left(\frac{\pi}{2}+x\right)=\sin \left(\frac{\pi}{2}\right) \cos (x)+\cos \left(\frac{\pi}{2}\right) \sin (x)=\cos (x)
\end{gathered}
$$

The same way,

$$
\sin \left(\frac{3 \pi}{2}-x\right)=-\cos (x) \sin \left(\frac{3 \pi}{2}+x\right)=-\cos (x)
$$

\section{Definition 2 (Functional extension).}

Be

$$
\begin{gathered}
F(x) / F: \mathbb{R} \rightarrow \mathbb{R} \\
F_{e}(x, y) / F_{e}: \mathbb{R}^{2} \rightarrow \mathbb{R}
\end{gathered}
$$

is a functional extension of $F(x)$ if.

$$
\exists y_{0} \in \mathbb{R}: F(x)=F_{e}\left(x, y_{0}\right)
$$

Property 1 (Derivative or zero order).

$$
\frac{\partial^{0} F(x)}{\partial x^{0}}=F(x)
$$

Proof of property 1. 
Using Definition 1 and $q=0$, the property is proved.

Definition 3 (Analytical-dimensional extension or a-d extension).

$$
F_{a}: \mathbb{R}^{2} \rightarrow \mathbb{R} / F_{a}(x, q)=\frac{\partial^{q} F(x)}{\partial x^{q}}
$$

is called a-d extension of $F(x)$. And it is a special case of functional extension because:

$$
F_{a}(x, 0)=F(x) \quad(\text { by Property } 1)
$$

i.e.:

$$
(x, q)^{2}=\frac{\Gamma(3)}{\Gamma(3-q)} x^{2-q}
$$

Definition 4 (Alternate a-d extension of sine function).

Using the ordinary derivative definition and basic properties of sine function:

$$
\text { easily } \frac{\partial^{q} \sin (x)}{\partial x^{q}}=\sin \left(x+\frac{\pi}{2} q\right)
$$

where $q \in \mathbb{N}$. So using a process of generalization to $q \in \mathbb{R}$

$$
\frac{\partial^{* q} \sin (x)}{\partial x^{q}}=\sin \left(x+\frac{\pi}{2} q\right)
$$

where $q \in \mathbb{R}$.

Principle 1 (Equivalence of $\frac{\partial^{q} \sin (x)}{\partial x^{q}}$ and $\frac{\partial^{* q} \sin (x)}{\partial x^{q}}$ ).

We take as true that:

$$
\frac{\partial^{q} \sin (x)}{\partial x^{q}}=\frac{\partial^{* q} \sin (x)}{\partial x^{q}}
$$

Given particular values $x=x_{0}$ and $q=q_{0}$. You can compute both a-d extensions and it is verifiable the coincidence of both outcomes.

Definition 5 (Ind-series).

$$
I S(x)=\lim _{n \rightarrow \infty} \sum_{i=0}^{n}(-1)^{i} \frac{0^{2 i+1-\frac{2 x}{\pi}}}{\Gamma\left(2 i+2-\frac{2 x}{\pi}\right)}
$$

It is called ind-series.

Theorem 1 (the ind-series Theorem).

Every real number $y$ is expressible as a reciprocal value of ind-series $I S\left(x_{i}+2 n \pi\right)$ and IS $\left(x_{j}+2 n \pi\right)$ where $n \in \mathbb{N}_{0}$, whose core domain is $[0,2 \pi]$ and $x_{i}$ and $x_{j}$ are symmetric respect to the ordinates $\frac{\pi}{2}$ if $x_{i}$ and $x_{j}$ are in the $[0, \pi)$ interval and $\frac{3 \pi}{2}$ if both of them are in the $[\pi, 2 \pi]$ interval.

Proof of Theorem 1.

$$
\sin (x, 0)=\sin (x) \quad \text { (by Property } 1)
$$




$$
\begin{aligned}
& \sin (x)=\sin (0+x) \\
& \sin (0+x)=\sin \left(0+\frac{\pi}{2}\left(\frac{2 x}{\pi}\right)\right) \\
& \sin \left(0+\frac{\pi}{2}\left(\frac{2 x}{\pi}\right)\right)=\sin \left(0, \frac{2 x}{\pi}\right) \text { (by Definition } 3 \text { and Principle 1) } \\
& \Leftrightarrow \sin (x, 0)=\sin \left(0, \frac{2 x}{\pi}\right) \\
& y=\sin (x)=\lim _{n \rightarrow \infty} \sum_{i=0}^{n}(-1)^{i} \frac{x^{2 i+1}}{\Gamma(2 i+1)} \\
& \Leftrightarrow \quad=\lim _{n \rightarrow \infty} \sum_{i=0}^{n}(-1)^{i} \frac{1}{\Gamma\left(2 i+2-\frac{2 x}{\pi}\right)} 0^{2 i+1-\frac{2 x}{\pi}}=I S(x) \quad \text { (By Definition 5) } \\
& y=\sin \left(\frac{\pi}{2}-\alpha\right)=\sin \left(\frac{\pi}{2}+\alpha\right) \vee y=\sin \left(\frac{3 \pi}{2}-\alpha\right)=\sin \left(\frac{3 \pi}{2}+\alpha\right) \text { (By lemma 2) } \\
& \sin : \mathbb{R} \rightarrow[0,1] \text { so } y \in[0,1] . \\
& \text { Be } I(x)=\left\{\begin{array}{ll}
\frac{1}{x}-1 & x \in[0,1] \\
\frac{1}{x}+1 & x \in[-1,0)
\end{array}\right. \text {. } \\
& \text { Then } \sin (o) I: \mathbb{R} \rightarrow \mathbb{R} .
\end{aligned}
$$

The steps above prove the Theorem.

\subsection{Analysis of Terms in Series of Indeterminate Values}

If $2 i+1-\frac{2 x}{\pi}<0$ then the general term is $(-1)^{i} \alpha_{i} \infty$

If $2 i+1-\frac{2 x}{\pi}=0$ then the general term is $(-1)^{i} \alpha_{i} 0^{0}$

If $2 i+1-\frac{2 x}{\pi}>0$ then the general term is $(-1)^{i} \alpha_{i} 0$

where $\alpha_{i}=\frac{\Gamma(2 i+2) \Gamma(2 i+1)}{\Gamma\left(2 i+2-\frac{2 x}{\pi}\right)}$.

So at first sight you probably observe that, for instance

$$
-\infty+\infty-\infty+\infty+0^{0}+0+0+0+\cdots=y_{i},
$$

where $y_{i} \in \mathbb{R}$.

It seems to break the axioms of arithmetic, but you have to take into account that you are operating with infinite amount of terms.

Besides, you have to make another analysis on the set of real numbers where the $\Gamma$ function is not defined. 
Remember that the indeterminate are:

$$
0^{0}, \infty-\infty, 0 \infty, \frac{0}{0}, \frac{\infty}{\infty}, \infty^{0}
$$

\subsection{Components of the Series}

Based on Section 7, we can express the ind-series to its eventual and deeper analysis as follows:

$$
I S(x)=I S_{\text {inf }}(x)+I S_{\text {indet }}(x)+I S_{\text {zero }}(x)
$$

where:

$I S_{\text {inf }}(x)$ is the partial sum where $2 i+1-\frac{2 x}{\pi}<0 \quad(\infty$ component $)$

$I S_{\text {indet }}(x)$ is the partial sum where $2 i+1-\frac{2 x}{\pi}=0 \quad\left(0^{0}\right.$ component $)$

$I S_{\text {zero }}(x)$ is the partial sum where $2 i+1-\frac{2 x}{\pi}>0 \quad(0$ component $)$

Therefore we can offer a notation for real numbers, called trienial.

Be $y_{0} \in \mathbb{R}$ then: $y_{0}$ could be expressible for the following possibilities:

$$
\begin{gathered}
\alpha \boldsymbol{i} \\
\beta \boldsymbol{j} \\
\gamma \boldsymbol{k} \\
\alpha \boldsymbol{i}+\beta \boldsymbol{j} \\
\beta \boldsymbol{j}+\gamma \boldsymbol{k} \\
\alpha \boldsymbol{i}+\gamma \boldsymbol{k} \\
\alpha \boldsymbol{i}+\beta \boldsymbol{j}+\gamma \boldsymbol{k}
\end{gathered}
$$

where $\boldsymbol{i}$ represents the $\infty$ components, $\boldsymbol{j}$ the $0^{0}$ component, and $\boldsymbol{k}$ the 0 component. And $\alpha, \beta, \gamma$ belongs to $\mathbb{R}$.

\subsection{A Last Point to Analyze}

Perhaps an issue pending analysis is to investigate the correspondence between a polynomial series $P(x)$ and a polynomial series $Q\left(x^{f(x)}\right)$.

In this case we can carry out a similar analysis as Section 6.

$$
\begin{aligned}
& \sin (x, 0)=\sin (x)=\sin \left(\frac{x}{2}+\frac{x}{2}\right)=\sin \left(\frac{x}{2}+\frac{\pi}{2} \frac{x}{\pi}\right)=\sin \left(\frac{x}{2}, \frac{x}{\pi}\right) \\
& \lim _{n \rightarrow \infty} \sum_{i=0}^{n}(-1)^{i} \frac{x^{2 i+1}}{\Gamma(2 i+2)}=\lim _{n \rightarrow \infty} \sum_{i=0}^{n}(-1)^{i} \frac{\left(\frac{x}{2}\right)^{2 i+1-\frac{x}{\pi}}}{\Gamma\left(2 i+2-\frac{x}{\pi}\right)}
\end{aligned}
$$

\subsection{Example of the Method}

$$
\sin \left(\frac{\pi}{2}\right)=1
$$




$$
\begin{aligned}
1 & =I S\left(\frac{\pi}{2}\right) \\
& =\lim _{n \rightarrow \infty} \sum_{i=0}^{n}(-1)^{i} \frac{0^{2 i}}{\Gamma(2 i+1)} \\
& =\lim _{n \rightarrow \infty} \sum_{i=0}^{n}(-1)^{i} \frac{0^{2 i}}{(2 i) !} \\
& =1 \frac{0^{0}}{1}+(-1) \frac{0}{2}+(1) \frac{0}{24}+\cdots \\
& =\lim _{n \rightarrow \infty} \sum_{i=0}^{n}(-1)^{i} \frac{\frac{\pi^{2 i+1}}{2}}{\Gamma(2 i+2)} \\
& =\lim _{n \rightarrow \infty} \sum_{i=0}^{n}(-1)^{i} \frac{\frac{\pi^{2 i+1}}{2}}{\Gamma(2 i+1) !}
\end{aligned}
$$

\subsection{Conclusion}

The facts that this class of series is not directly computational and any convergence criterion cannot be applied to them, stand out and give relevance to those above mentioned theorems.

The approaches offered by the non-standard analysis and the way it is showed to operate with the "unbounded" and "infinitesimal" numbers do not lead to some outcome [3].

\section{Conflicts of Interest}

The author declares no conflicts of interest regarding the publication of this paper.

\section{References}

[1] Guia Calderon, M., et al. (2015) El cálculo diferencial e integral fraccionario y sus aplicaciones. Acta Universitatis, 25, No. 2. http://www.scielo.org.mx/scielo.php

[2] Brown, J. and Churchill, R. (1989) Complex Variables and Applications. McGraw-Hill, New York.

[3] Robert, A. (1988) Nonstandard Analysis. Wiley, New York. 